\title{
Optimal Wheelchair Passenger Service in the Airport
}

\author{
Anran Zheng \\ School of North China Electric Power University Baoding, Baoding 071000, China; \\ zhenganran1995@126.com
}

Keywords: Queuing theory, Multi-server system, Optimization

\begin{abstract}
This paper uses a queuing model of multi-server system to offer optimal airport wheelchair service which minimizes the cost and offers service of high quality. It addresses the performance of airport wheelchair service associated with the waiting time of passengers who need the service. According to the related data, we can know the cost of wheelchair service in unit time $C_{s}$ and the loss of keeping passengers waiting for service in unit time $C_{w}$. Basing on the related equations of queuing theory, we calculate the ratio between $C_{s}$ and $C_{w}$ and compare it with numerical interval in different number of the wheelchairs, thus we get the optimal number of wheelchair and the cost. We take the O'Hare International Airport as an example and calculate the optimal wheelchair passenger service. This model can be applied to airports of different shapes and sizes.
\end{abstract}

\section{Introduction}

For airports, offering good service to passengers in the air travel is extremely important ${ }^{[1]}$. However one of the frustrations with air travel is the need to fly through multiple airports, and each stop generally requires each traveler to change to a different airplane. This is inconvenient and difficult to people who are not able to walk such as disables and the elderly. They may need wheelchair service from the airport. Wheelchair passenger service(wps) is providing a wheelchair and an escort to those people who ask for help. It is generally known well in advance which passengers require help. But in rare instances the airport may not receive notice until the passenger lands. On this issue, the cost is also very important which includes the escorts' salaries and the maintenance cost of wheelchairs. One of the biggest costs is the cost of holding a plane if someone must wait for an escort and becomes late for their flight.

To solve the problem, we set up a queuing model of multi-server system to offer optimal wps which minimizes the cost and offers service of high quality.

\section{Queuing Model of Multi-server System}

Queuing theory. Queuing theory is familiar to us. Due to the randomness of the customers' arrival and service time, queuing phenomenon is almost inevitable. Service system design often considers the balance between the service cost and expected customer waiting costs in the system. In general, the service cost is increasing function of the service level. The improvement of the service level will reduce customer's waiting costs, but it often increases the cost of service. Optimization goal is to make both the total cost minimum, corresponding to the most proper number of the service system.

Analysis of Multi-server System. In our model, those who need wps are "customers" and the escort who has a wheelchair is a "service system". The queuing system is of single line multi-server system $^{[2]}$. According to the queuing theory, the model is $\mathrm{E}_{2} / \mathrm{M} / \mathrm{c} / \infty / \infty$.

(1). Arrival process obeys the Erlang-2 distribution. The probability density function of Erlang-2 distribution is: 


$$
p_{1}(t)= \begin{cases}\frac{\mu \cdot\left(\mu t^{k-1}\right)}{(k-1) !} e^{-\mu t} & t \geq 0 \\ 0 & t<0\end{cases}
$$

Where $\mu$ is the average time of customers' arrival, $k$ is the number of stage $^{[3]}$. It can be interpreted as customers must pass a barrier which constitute of $k$ layers to get the window to line up. The time of passing each layer obeys negative exponential distribution with parameters $\mu k$.

In our model, $\mathrm{k}$ is 2 . There are two layers of barrier in the way to the window of line. In the first layer, because the percentage of people who need wps is a fixed value $q$. Once the passenger reaches the terminal, there is a judgment of binomial distribution on whether he needs service. According to the law of large numbers, there are lots of people arriving at the airport in a day, so binomial distribution can be approximately the Poisson distribution. We use backstage to define the first layer.

The second layer is the process of picking the customers for escorts. Once the customer reaches the terminal, the airport controller can know his position at once and issue a directive to the escort who can help the customers in the shortest time by radio. The escorts in the airport distribute uniformly in time and space. The probability on whether there is an escort can offer help in the corresponding time is equal. So the layer obeys the Poisson distribution like the first one. The Erlang-2 distribution is two identical superposition of Poisson distribution. So the arrival process obeys Erlang-2 distribution.

(2). Service process obeys the Poisson distribution.

Negative exponential distribution is the same with Poisson distribution but in different expression form. The probability density function of negative exponential distribution is:

$$
p_{2}(t)= \begin{cases}\mu \cdot e^{-\mu t} & t \geq 0 \\ 0 & t<0\end{cases}
$$

Where $\mu$ is the average time of service time. The whole process begins when the customer accepts wps and stops when he is delivered to the aircraft by the escort.

(3). Queuing rule is that first come first service with the system capacity infinite.

Under normal circumstances, the capacity of the airport and the number of customer source is approximately infinite. To demonstrate the whole process better, we have figure 1. In figure 1, $\lambda=1 / \mu$ where $\lambda$ is the number of customers in unit time. We use $c$ to define the size of service system.

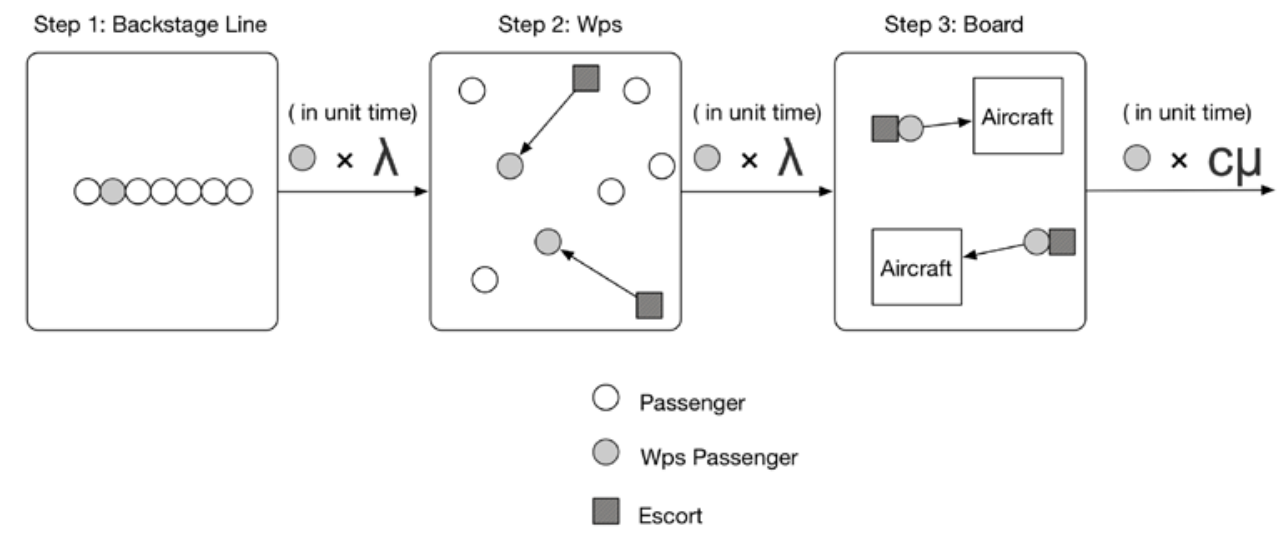

Figure 1 the normal queuing process

The $\lambda$ is equal in the first two steps, because if the $\lambda$ is not equal it will cause congestion and idleness in different step which is extremely adverse for airport operation.

Optimal number of service system. We can get the probability density function of input process system 


$$
p_{1}(t)= \begin{cases}\alpha^{2} t e^{-\alpha t} & t \geq 0 \\ 0 & t<0\end{cases}
$$

and the probability density function of service time

$$
p_{2}(t)= \begin{cases}\beta e^{-\beta t} & t \geq 0 \\ 0 & t<0\end{cases}
$$

Therefore, from equation (3)(4), we have $\mu=1 / \beta$ and $\lambda=\alpha / 2$ where $\lambda$ is the average arrival rate and $\mu$ is the average service time per person. We use $\rho$ to define the system service intensity: $\rho=\lambda / c \mu$. The probability of system idle is

$$
P_{0}=\left[\sum_{k=0}^{c-1} \frac{1}{k !}\left(\frac{\lambda}{\mu}\right)+\frac{1}{c !} \cdot \frac{1}{1-\rho} \cdot\left(\frac{\lambda}{\mu}\right)^{c}\right]^{-1}
$$

The average number of customers in the queue is

$$
L_{q}=\sum_{m=c+1}^{\infty}(n-c) P_{n}=\frac{(c \rho)^{c} \rho}{c !(1-\rho)^{2}} P_{0}
$$

The expectations of queue length is

From Little’s law, we have

$$
L_{s}=L_{q}+\frac{\lambda}{\mu}
$$

$$
W_{q}=\frac{L_{q}}{\lambda}, W_{s}=\frac{L_{s}}{\lambda}
$$

Where $W_{q}$ is the average waiting time, $W_{s}$ is the average sojourn time.

For $\mathrm{E} 2 / \mathrm{M} / \mathrm{c} / \infty / \infty$ system, the expectation of the whole cost in unit time is

$$
z=C_{s} \cdot c+C_{w} \cdot L
$$

Where $C_{s}$ is the total cost of each wheelchair and its corresponding escort in unit time, $C_{w}$ is the cost of the customers' waiting time. We can know $C_{w}, C_{s}$ by querying the relative airline data. Because $C_{w}, C_{s}$ are known, $z$ is the function of $z(c)$. So we find the optimal $c^{*}$ to make $z\left(c^{*}\right)$ minimum. According to the characteristic of $z\left(c^{*}\right)$ which is the minimum, we use marginal analysis and get

$$
z\left(c^{*}\right) \leq z\left(c^{*}-1\right), z\left(c^{*}\right) \leq z\left(c^{*}+1\right)
$$

Putting (9) into (10), we have

$$
L\left(c^{*}\right)-L\left(c^{*}+1\right) \leq \frac{c_{s}}{c_{w}} \leq L\left(c^{*}-1\right)-L\left(c^{*}\right)
$$

Then we respectively calculate the value of $L$ in $c=1,2,3, \ldots$, and calculate the difference of adjacent $L$. Because, $C_{s} / C_{w}$ is a constant, we can get the optimal number of service system $c^{*}$ by judging which interval $C_{s} / C_{w}$ belongs.

Numerical Results in O'Hare. Chicago O'Hare International Airport(O'Hare) is an international airport located on the Far Northwest Side of Chicago ${ }^{[4]}$. It is the world's busiest airport in number of takeoffs and landings. According to the data we collected, we have $\lambda=104.5, \mu=1, C_{w}=3.4$ per minute. Because the lack of data, we make some assumptions in the process which is acceptable. But the airline can find the data easily. We assume the salary of escort is 3000 dollars per month(i.e.100 dollars per day). The wheelchair can work for 24 hours but the man can't. We assume three escorts take turn to work in 24 hours so the cost is 300 dollars per day(i.e.0.21 dollar per minute). The wear cost of each wheelchair is 80 dollars per year(i.e. 0.00015 per minute), which is too small that can be ignored. So we have $C_{s} / C_{w}=0.0617$. According to (11), we respectively calculate the value of $L$ in $c=90,91, \ldots$ 
Table 1 the value of $L\left(c^{*}\right)$ and $L\left(c^{*}-1\right)-L\left(c^{*}\right)$ in different value of $c^{*}$

\begin{tabular}{lcccccccc}
\hline$c^{*}$ & 105 & 106 & 107 & 108 & 109 & 110 & 111 & 112 \\
$L\left(c^{*}\right)$ & 196.785 & 57.984 & 30.633 & 19.188 & 13.032 & 9.271 & 6.790 & 5.070 \\
$L\left(c^{*}-1\right)-L\left(c^{*}\right)$ & 0.000 & 138.800 & 27.351 & 11.445 & 6.156 & 3.761 & 2.481 & 1.720 \\
\hline$c^{*}$ & 113 & 114 & 115 & 116 & 117 & 118 & 119 & 120 \\
$L\left(c^{*}\right)$ & 3.836 & 2.929 & 2.250 & 1.736 & 1.342 & 1.039 & 0.804 & 0.622 \\
$L\left(c^{*}-1\right)-L\left(c^{*}\right)$ & 1.234 & 0.907 & 0.679 & 0.515 & 0.394 & 0.303 & 0.235 & 0.182 \\
\hline$c^{*}$ & 121 & 122 & 123 & 124 & 125 & 126 & 127 & 128 \\
$L\left(c^{*}\right)$ & 0.480 & 0.370 & 0.284 & 0.218 & 0.166 & 0.127 & 0.096 & 0.072 \\
$L\left(c^{*}-1\right)-L\left(c^{*}\right)$ & 0.142 & 0.110 & 0.086 & 0.066 & 0.051 & 0.040 & 0.031 & 0.024 \\
\hline
\end{tabular}

From the table above, we can find 0.0617 is in the interval 0.051-0.066. So we find the optimal value of $c=124$ which means that the O'Hare needs 124 wheelchairs and corresponding escorts.

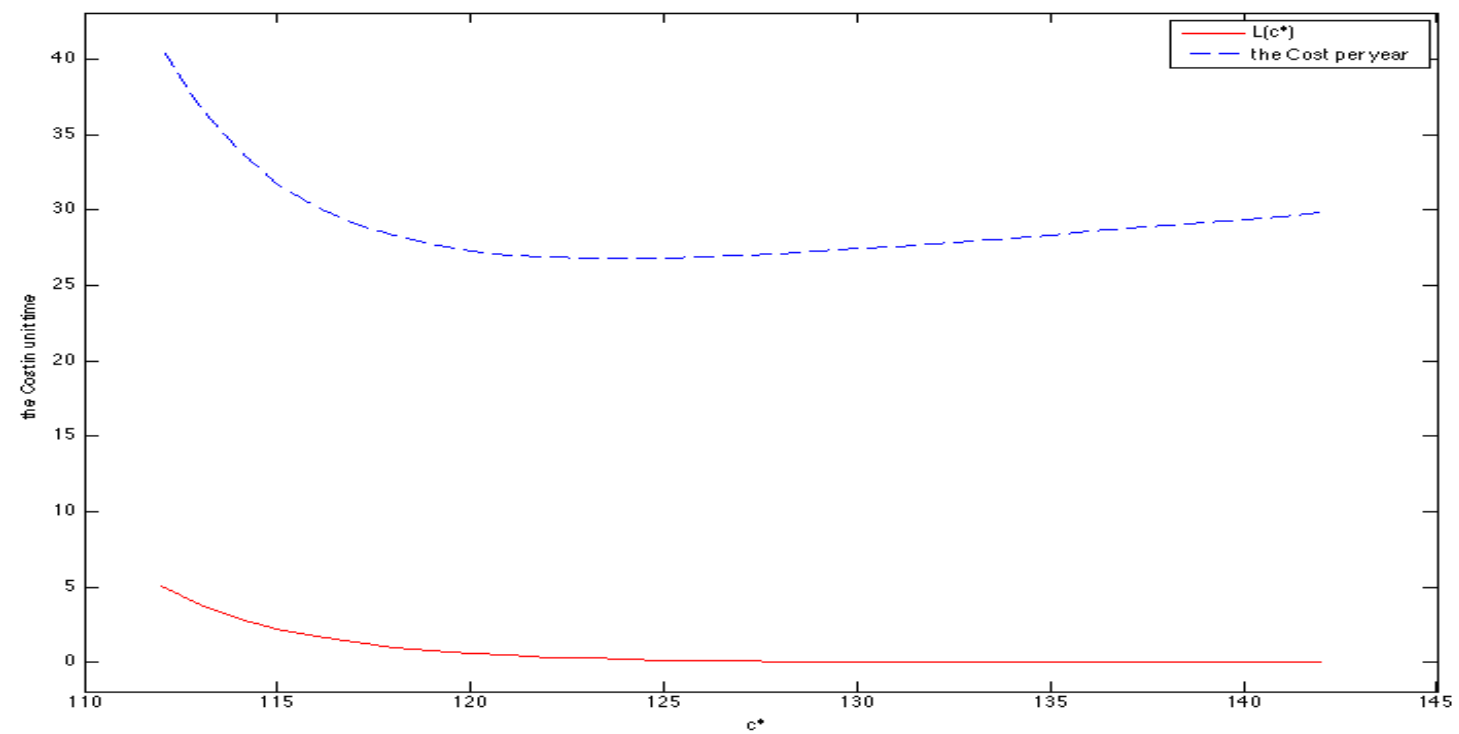

Figure 2 the cost in unit time in different value of $c^{*}$

From figure 2, we can see the cost in unit time in different value of $c^{*}$, which shows we find the optimal number of wheelchair and makes the cost minimum. Thus the whole cost of the service is 26.78 dollar per minute. So the cost of O'Hare on this service is 38563 dollars in a day.

\section{Summary}

In our paper, we build the optimal queuing model of multi-server system to minimize the cost on the wheelchair service. We consider the impact of time on the demand of service regardless of the shape of the airport. Therefore, the model can be applied to airports of different shapes and sizes. With the increasing of aging population, the need of the wheelchair service is also increasing, we just need to improve the proportion of the customers in the passengers then we can predict increment of wheelchair and corresponding escort and the cost of the airport in the future.

\section{References}

[1]http://www.iata.org/Pages/default.aspx

[2] Ning Cui, Jiang Han, Wei Zheng. The Optimal Analysis of the Queue Model of More Service Sytem. www.paper.edu.cn/download/downPaper/201012-334

[3] Erlang distribution. http://en.wikipedia.org/wiki/Erlang_distribution

[4]O'Hare International Airport. http://en.wikipedia.org/wiki/O\%27Hare_International_Airport 
[5] Sheng Z, Xie S, Pan C. Probability theory and mathematical statistics.China Higher Education Press: Beijing, 2001: 129-131. 